\title{
Estrogen supplementation failed to attenuate biochemical indices of neutrophil infiltration or damage in rat skeletal muscles following ischemia
}

\author{
PETER M. TIIDUS, MIRADA DELLER, ERIC BOMBARDIER, MUSTAFA GÜL \\ and X. LINDA LIU
}

Department of Kinesiology and Physical Education, Wilfrid Laurier University, Waterloo Ontario, Canada.

\begin{abstract}
This study examined the effects of estrogen supplementation on markers of neutrophil infiltration and damage in skeletal muscle of rats following ischemia. Male and female gonad-intact rats, with or without 14 days of estrogen supplementation were subjected to two hours of hind-limb ischemia and sacrificed at 24, 48 or 72 hours post-ischemia. Control animals were sacrificed without ischemia. Plantaris and red and white gastrocneimus muscles were removed and assayed for myeloperoxidase (MPO), a marker of neutrophil infiltration, and glucose-6-phosphate dehydrogenase (G6PD) and ß-glucuronidase ( $\exists G L U)$, as markers of muscle damage. Significant elevations of MPO, G6PD and $\exists$ GLU activities were observed at various time points post-ischemia. No systematic differences between genders were noted in any of the measures. Estrogen supplementation in both male and female animals failed to significantly attenuate post-ischemia increases in MPO, G6PD and $\exists$ GLU activities in any of the muscles studied and in some cases accentuated activities of some of these measures. Unlike previous findings following exercise in skeletal muscle, this study failed to demonstrate estrogen-induced attenuation of indices of neutrophil infiltration or damage in skeletal muscles of rats up to 72 hours following ischemia. This demonstrates that estrogen may not consistently attenuate neutrophil infiltration and that a number of variables including damage modality, tissue or estrogen level may influence this.
\end{abstract}

Key terms: ischemia, estrogen, muscle damage, skeletal muscle, neutrophils.

\section{INTRODUCTION}

Prolonged ischemia in skeletal muscle followed by reperfusion results in edema, necrosis, inflammation, leukocyte infiltration and damage (Rubin et al., 1996). Neutrophil infiltration is generally accepted to be an integral component of tissue inflammatory response to damaging insult and neutrophils may be able to influence both post-injury muscle damage and repair responses to varying degrees (Pizza et al., 2002; Schlag et al., 2001).

We have previously reported that estrogen supplementation in male and overiectomized female rats is able to attenuate indices of neutrophil accumulation in skeletal muscle following running exercise for up to 24 hours (Tiidus \& Bombarider 1999; Tiidus et al., 2001). The mechanisms by which estrogen can influence post-damage leukocyte accumulation in skeletal muscle are not yet fully defined but may be related to its ability as a membrane stabilizer and antioxidant or its influence on other estrogen receptor or non-receptor mediated mechanisms (Tiidus, 2003; Bär \& Amelink, 1997; Kendall \& Eston, 2002; Nilsson et al., 2001). It has been suggested recently that various forms of muscle damage may activate different regulatory or leukocyte recruitment mechanisms and thereby influence the quality and/or quantity of post-insult leukocyte infiltration into skeletal muscle (Lapointe et al., 2002; Pizza et al., 2002). It is therefore possible that the

Correspondence to: Peter M. Tiidus, Ph.D., Professor \& Chair, Department of Kinesiology \& Physical Education, Wilfrid Laurier University, 75 University Ave W., Waterloo, Ontario, Canada, N2L 3C5, Tel.: (1-519) 884-0710, ext. 4157, Fax: (1-519) 747-4594,E-mail: ptiidus@wlu.ca 
influence of estrogen supplementation on post-ischemic leukocyte infiltration into skeletal muscle may differ from its influence following running exercise in rats.

Relatively few studies have examined the potential for estrogen to influence skeletal muscle neutrophil infiltration following ischemia-reperfusion induced injury. Using cardiac muscle, Squadrito et al. (1997) reported that estradiol-17b supplementation diminished immediate neutrophil accumulation, as determined by myeloperoxidase activity in female rats immediately after reperfusion of the ischemic myocardium. Only one previous study has examined the potential for estrogen to influence neutrophil infiltration following ischemia in skeletal muscle. This study reported attenuation of indices of neutrophil infiltration, as indicated by smaller elevations in CD43+ cells, in skeletal muscle two hours following ischemia with estrogen supplementation in ovariectomized female rats relative to controls (Stupka and Tiidus, 2001). However, no information is currently available regarding the potential influence of estrogen supplementation on neutrophil infiltration following ischemia beyond two hours of reperfusion.

Indices of skeletal muscle damage also may be influenced by estrogen or gender (Amelink et al.,1991; Komulainen et al., 1999). Ischemia-reperfusion induced damage and post-injury recovery in cardiac muscle has been reported to be improved by estrogen supplementation in most (i.e., Node et al., 1997; Squadrito et al., 1997; Zhai et al., 2000) but not all (Grist et al., 2002) studies. No previous studies have examined the potential for estrogen supplementation to influence indices of muscle damage following ischemiareperfusion injury in skeletal muscle.

Previous studies also usually have not considered potential muscle fiber type differences for estrogen influence on the extent of insult-induced damage or muscle neutrophil infiltration (Stupka \& Tiidus, 2001). Estrogen receptor presence and density in skeletal muscle of rats may be influenced by gender, training or circulating estrogen levels (Lemoine et al., 2002).
However, little is known about potential differences in estrogen receptor densities in muscles of differing fiber populations or of the potential for these differing muscles to be influenced by estrogen supplementation.

This study examined the potential for estrogen supplementation to influence biochemical indices of neutrophil infiltration and damage in muscles of differing fiber type populations from gonad-intact male and female rats for up to 72 hours following ischemic insult.

\section{METHODS}

\section{Animals}

This study was approved by the Wilfrid Laurier University Committee on Animal Care as being in compliance with the guiding principles for the use of animals in research as outlined by the Canadian Council on Animal Care. Gonad-intact male and female Sprague-Dawley rats (Charles River Quebec, Canada) were used in the study. Rats were housed by gender with 2 animals per cage prior to the ischemia procedure and individually post-ischemia. The rats were kept in an environmentally controlled room with reversed 12:12 light/ dark cycles and allowed free access to food (Teklad 22/5 Rodent Diet, HarlandTekland) and water. The rats were 8 weeks old upon arrival to our laboratory.

\section{Experimental design}

After 1 week of acclimation, rats were randomly assigned by gender to either the estrogen implant or sham implant group. All animals were placed under halothane anesthesia for subcutaneous implant of either a 21-day release estrogen pellet $(0.25 \mathrm{mg}$ bestradiol in vegetable oil vehicle) using a 10gauge needle or a sham implant (incision and needle insertion alone). All rats were handled and weighed daily for 2 weeks post-implant to allow for prolonged elevated estrogen exposure in the designated groups (Tiidus and Bombardier, 1999). Subsequently, rats were anaesthetized via interperitoneal injection of pentobarbitol sodium $(100 \mu / 100 \mathrm{~g} \mathrm{BW})$ 
(Somnatol, MTC Pharamceuticals, Cambridge, ON, Canada). Anesthesia was confirmed with the loss of withdrawal reflex from a toe web pinch and maintained by pentobarbitol sodium injections as required. Complete ischemia of the left hind limb was induced via small animal surgical tourniquet over the upper left hind limb for two hours after which the tourniquet was released. Preliminary observations indicated that complete ischemia was achieved with this technique as employed in this experiment. Animals were kept warm with a heating pad and vital signs monitored throughout the procedure. Animals were replaced in their cages, allowed to recover. At 24, 48 and 72 hours post-ischemia, male and female estrogen-supplemented and sham rats (approximately 10 per group) were again anesthetized with pentobarbitol sodium as described above. Control male and female estrogen-supplemented and sham rats (approximately 10 per group) were sacrificed without ischemia. Rats were approximately 12 weeks of age and sexually mature at the time of sacrifice.

The lower abdomen was opened and blood samples $(5 \mathrm{ml})$ were withdrawn from the descending aorta. Blood was allowed to clot, centrifuged for 10 minutes at $500 \mathrm{x} \mathrm{g}$ and the serum removed and stored at $-25^{\circ} \mathrm{C}$. Lower limb ankle extensor muscles were surgically removed, trimmed of visible connective tissue and tendons, rinsed in saline and blotted to remove any blood, quickly frozen in liquid nitrogen and stored at $-80^{\circ} \mathrm{C}$ until analysis. The muscles used in this study were plantaris (PL) (approximately 50\% type IIa, $40 \%$ type IIb and $10 \%$ type 1), and the lateral gastrocnemius which was visually separated into white-superficial (WG) (almost entirely type II b) and red-deep (RG) (approximately $80 \%$ type IIa and $20 \%$ type I) sections prior to storage (Ariano et al., 1973; Gillespie et al., 1987).

\section{Blood and tissue analysis}

Serum samples were analyzed for estradiol content using a commercially available RIA kit (\#TKE21, Diagnostic Products, Los Angeles, CA, USA) using 250 F L serum samples in duplicate (Tiidus et al., 2001). All muscle samples (PL, WG, RG) were analyzed for myeloperoxidase (MPO), glucose-6phosphate dehydrogenase (G6PD) and bglucuronidase (bGLU) activites. Muscle MPO activity is a marker of leukocyte (primarily neutrophil) infiltration (Belcastro et al., 1996). Muscle MPO activity was determined spectrophotometrically from homogenates using the method of Smith and colleagues (1989). One unit of MPO activity was defined as a change in one unit of abs at $480 \mathrm{~nm} \mathrm{~min}{ }^{-1}$ and expressed per $\mathrm{g}$ muscle wet weight (Belcastro et al., 1996). Muscle G6PD activity is a semi-quantitative marker of muscle damage (Armstrong et al., 1983). Muscle G6PD activity levels were determined fluorometrically from homogenate as described by Armstrong and colleagues (1983). G6PD activity was expressed as $\mu \mathrm{mol}$ NADPH generated mg protein ${ }^{-1}$ minute $^{-1}$ at 20EC. Protein was determined using the method of Lowery and colleagues (1951). Muscle bGLU activity is an indicator of muscle lysosomal enzyme activity and a reliable quantitative marker of muscle damage (Salminen and Kihlström, 1985). Muscle bGLU activity was determined spectrophotometrically from homogenates as described by Komulainen and colleagues (1999). bGLU activity was expressed as a change in one unit of abs at $420 \mathrm{~nm} \mathrm{mg}$ protein ${ }^{-1} \mathrm{~min}^{-1}$ at $20 \mathrm{EC}$.

\section{Statistical analysis}

Data are expressed as mean \pm S.D. Data analysis between control and 24, 48 and 72 hour post-ischemia time points within each gender and between estrogen-supplemented and sham groups for each muscle were performed by analysis of variance (ANOVA) with Tukey-Kramer multiple comparison post-hoc tests when a significant difference between groups was noted. Significance was defined as $\mathrm{p}<0.05$.

\section{RESULTS}

Female estrogen supplemented and sham rats weighed $237.5 \pm 15.2 \mathrm{~g}$ and $219.8 \pm 15.5 \mathrm{~g}$ respectively immediately prior to ischemia, while male estrogen-supplemented and sham 
rats weighed $350.1 \pm 14.8 \mathrm{~g}$ and $408.2 \pm 12.9$ $\mathrm{g}$ respectively. These weights were not significantly altered in these respective groups up to 72 hours post-ischemia. Averaged serum estradiol levels (pg mL) were significantly greater $(\mathrm{p}<0.001)$ in the estrogen-supplemented male and female rats $(649.5 \pm 49.6$ and $452.2 \pm 311.8$ respectively) than in the control male and female (sham) rats $(9.6 \pm 2.1$ and $24.2 \pm 11.6$ respectively). The estradiol levels in the represent peak physiological levels and were higher than used in some of our previous studies (i.e., Stupka and Tiidus, 2001). It was expected that as with some other aspects of estrogen influence on muscle, higher levels may exert a greater influence (Sarwar et al., 1996).

PL, WG and RG muscle MPO activity results for male and female estrogen-supplemented and sham rats are depicted in Figure 1. While the trend toward elevated MPO activities in the postischemic time points relative to control values for both male and female rats is evident, relatively few time points reached statistical significance. Interestingly, those post-ischemia time points in muscles that were significantly elevated relative to controls $(\mathrm{p}<0.01)$ (PL male 24-hours postischemia, WG male 48 -hours post-ischemia and female 24-hours post-ischemia) were in the estrogen-supplemented rats and also were significantly higher $(\mathrm{p}<0.05)$ in MPO activity than the corresponding time sham animals. There seemed to be no clear pattern in occurrence of elevated MPO activities in post-ischemia time-course or muscle.

PL, WG and RG muscle G6PD activity results for male and female estrogen-supplemented and sham rats are depicted in Figure 2. G6PD activities are significantly $(\mathrm{p}<0.05)$ elevated at 72 -hours post-ischemia in all of the muscles investigated in estrogen and sham compared to the non-ischemia exposed controls in both male and female rats (with the exception of the female WG sham group). Some of the 24- and 48-hour time points for male and female RG, PL and WG muscles also exhibit significantly elevated G6PD activities $(\mathrm{p}<0.05)$ relative to their respective controls. At no post-ischemia time point examined in either male or female rats does estrogen supplementation result in an attenuation of G6PD activity relative to sham rats in $R G, W G$ or PLA muscles. In fact, at 72 hours post-ischemia in male RG and female WG muscles, estrogen-supplemented animals exhibited significantly higher G6PD activities than sham rats at the equivalent time point. Nevertheless, as with MPO activities, estrogen supplementation did not appear to attenuate post-ischemic G6PD activities in RG, WG or PLA muscles of either male or female rats at any time up to 72 hours.

PL, WG and RG muscle bGLU activity results for male and female estrogen-supplemented and sham rats are depicted in Figure 3. bGLU activities are elevated significantly $(\mathrm{p}<0.05)$ in one or more post-ischemia time points relative to controls in PL, WG and RG muscles of both male and female rats. No clear differences exist between genders in PL, WG or RG muscle bGLU activities nor are there any significant effects of estrogen supplementation, relative to corresponding sham group on muscle bGLU activities at any post-ischemia time point.

\section{DISCUSSION}

Estrogen supplementation in male and female gonad-intact sexually mature rats failed to attenuate indices of neutrophil infiltration (as MPO activity) in muscle of primarily IIb (WG), IIa (RG) and mixed (PL) fiber types for up to 72 hours following two hours of ischemia. In fact, in some situations, estrogen-supplemented animals appeared to have augmented MPO activities following ischemia relative to sham animals. In addition, estrogen supplementation failed to influence elevations of G6PD or bGLU activity (as indicators of muscle damage) in $\mathrm{WG}, \mathrm{RG}$ or PL muscles relative to sham in either male or female rats for up to 72 hours following ischemia. There were no consistent gender differences noted in any of the results. These results are in contrast to the reported effectiveness of estrogen supplementation 

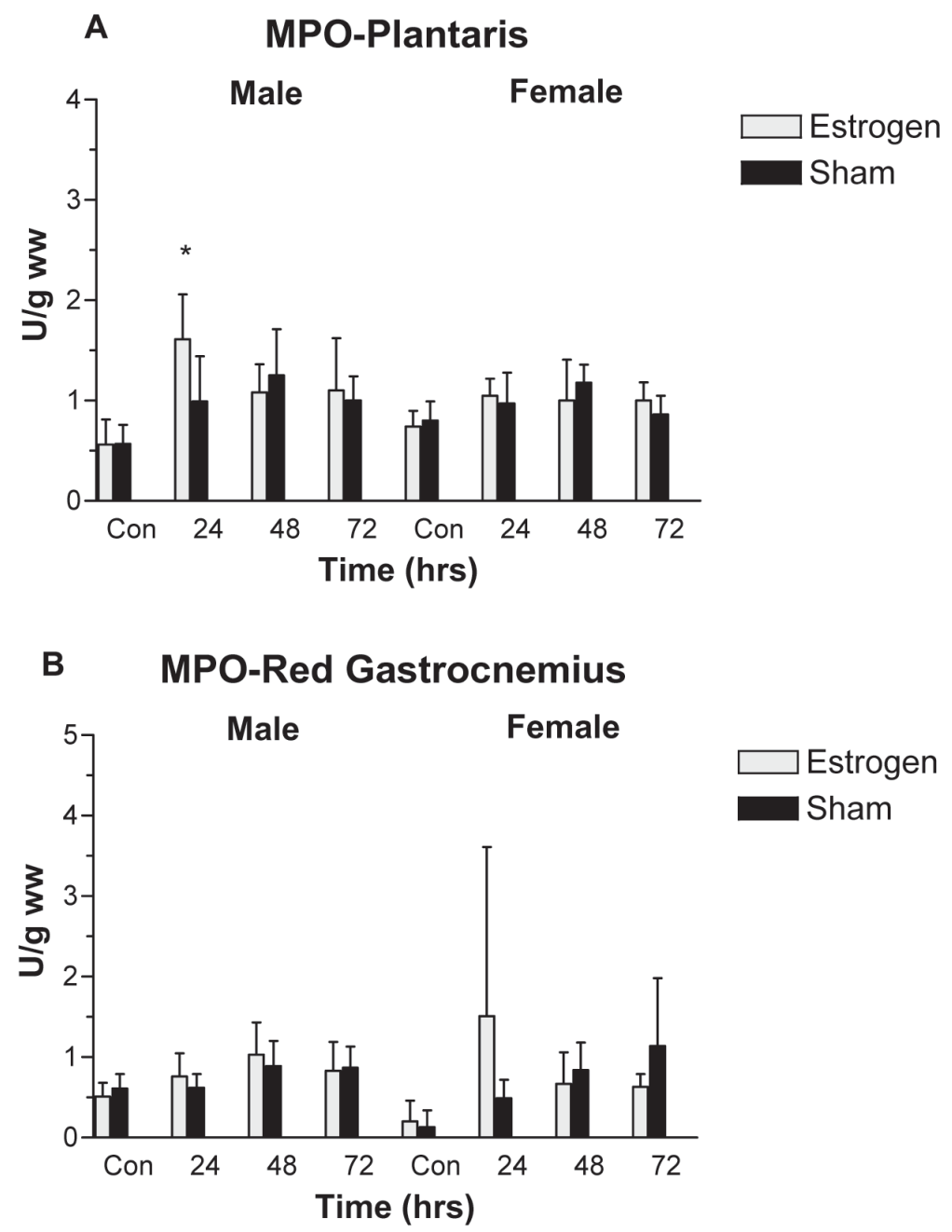

\section{MPO-White Gastrocnemius}

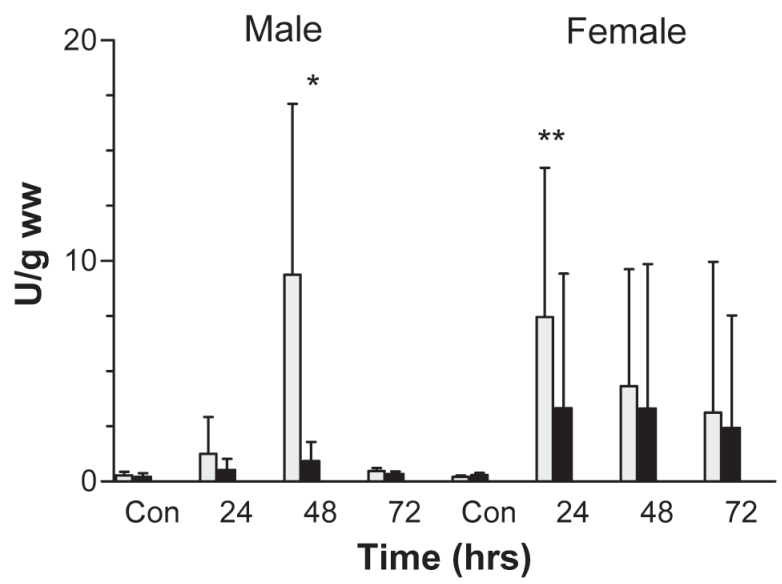

Figure 1. Myeloperoxidase (MPO) activities in plantaris, red gastrocnemius and white gastrocnemius muscles of male and female rats with estrogen supplementation or sham, at 24, 48 and 72 hours post- 2 hour ischemia or in control (con) animals. Open boxes = estrogen supplemented, filled boxes = sham, * greater than control $(\mathrm{p}<0.05),{ }^{* *}$ greater than sham and control $(\mathrm{p}<0.05)$. 

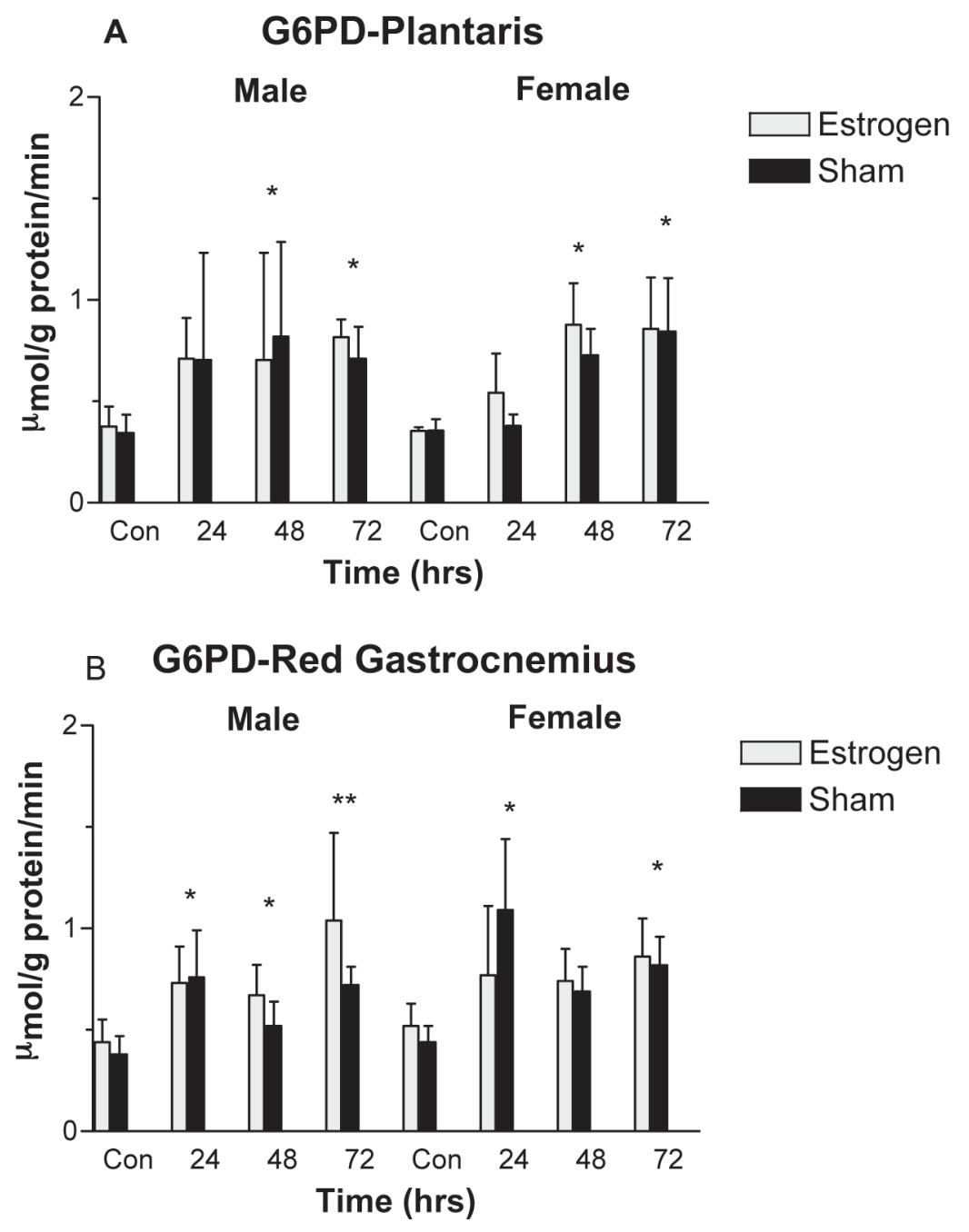

C G6PD-White Gastrocnemius

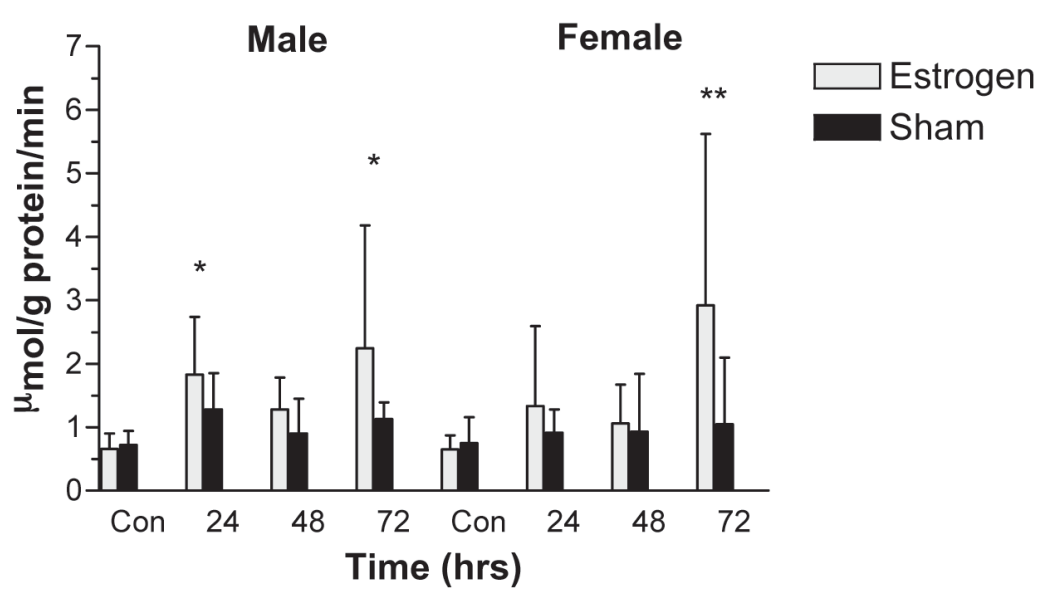

Figure 2. Glucose-6-Phosphate Dehydrogenase (G6PD) activities in plantaris, red gastrocnemius and white gastrocnemius muscles of male and female rats with estrogen supplementation or sham, at 24,48 and 72 hours post- 2 hour ischemia or in control (con) animals. Open boxes = estrogen supplemented, filled boxes $=$ sham, $*$ greater than control $(\mathrm{p}<0.05), * *$ greater than sham and control $(\mathrm{p}<0.05)$. 

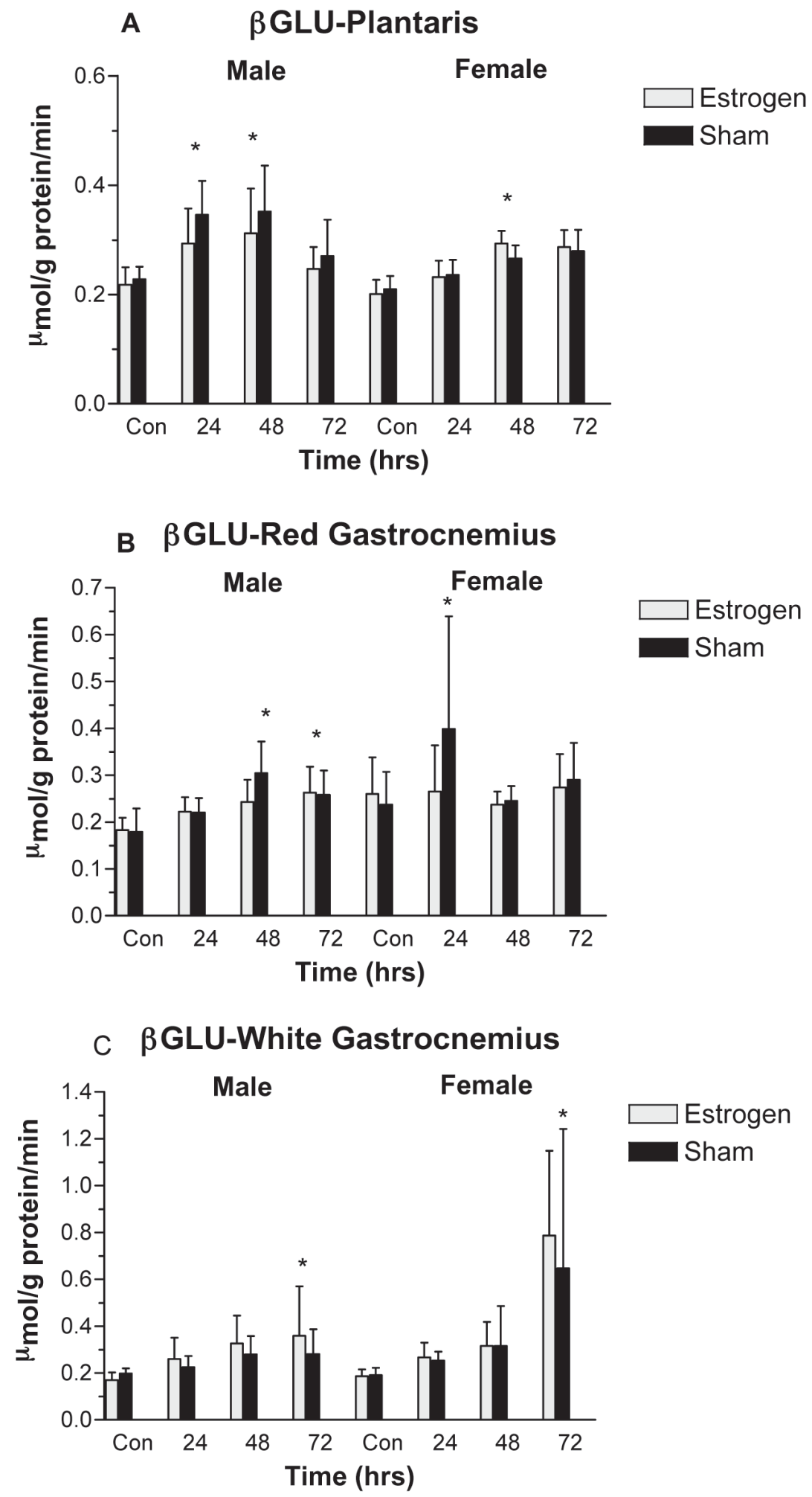

Figure 3. b-Glucuronidase (bGLU) activities in plantaris, red gastrocnemius and white gastrocnemius muscles of male and female rats with estrogen supplementation or sham, at 24, 48 and 72 hours post- $2 \mathrm{hr}$ ischemia or in control (con) animals. Open boxes = estrogen supplemented, filled boxes $=$ sham, ${ }^{*}$ greater than control $(\mathrm{p}<0.05)$. 
in attenuating indices of neutrophil infiltration following running exercise in male and ovariectomized female rats (Tiidus and Bombardier, 1999; Tiidus et al., 2001), and in the reported attenuation of indices of muscle damage and bGLU activity following downhill running in male versus female rats (Komulainen et al., 1999). The results also contrast most (i.e., Node et al., 1997; Squadrito et al., 1997; Zhai et al., 2000) but not all studies (Grist et al., 2002), which suggest a reduction on indices of neutrophil infiltration and damage with estrogen supplementation following ischemia in cardiac muscle.

The reasons for the lack of positive influence of estrogen supplementation on post-ischemic muscle neutrophil infiltration and indices of muscle damage are not immediately clear but do suggest that it is not universally present and that a number of factors present in this study might alter this effect.

In this study, muscle MPO activity was used as an indicator of neutrophil infiltration. We previously had reported significant attenuation of increases in CD43+ stained cells in soleus muscle of ovariectomized female rats supplemented with estrogen relative to shamsupplemented animals at 2 hours following ischemia (Stupka and Tiidus, 2001). The presence of CD43 has been used as an indicator on neutrophil infiltration of skeletal muscle following damage (Tidball et al., 1999). Muscle MPO activity also has been commonly used as a quantitative marker of muscle neutrophil infiltration following exercise or ischemia induced damage (i.e., Squadrito et al., 1997; Belcastro et al., 1996; Tiidus et al., 2001). Our previous study found no elevation in MPO activities in soleus muscle at two hours after a two-hour ischemic period in ovariectomized or gonad-intact female rats (Stupka and Tiidus, 2001). However, others have reported significant elevations in muscle MPO activities at one hour following a four-hour period of ischemia in dogs (Smith et al., 1989). It is possible that 3-5 hours of ischemia is necessary to induce consistent elevations in muscle MPO activities following reperfusion (Schlag et al., 2001). Nevertheless, where the twohour ischemia employed in this study did induce significant post-ischemic elevations in muscle MPO activities, these were more evident in the estrogen-supplemented rats than in shams, particularly in predominantly type II muscle.

A wide variety of factors including oxygen radicals, various cellular adhesion factors, pro-inflammatory cytokines and others may directly or indirectly influence cellular neutrophil infiltration (Rubin et al., 1996). The mechanisms by which estrogen may influence post-damage muscle neutrophil infiltration are not known. Few studies have directly investigated the mechanisms of estrogen influence on postischemic skeletal muscle neutrophil infiltration. However post-ischemic effects of estrogen on cardiac neutrophil infiltration may be mediated through its potential influence on intercellular adhesion molecule-1 (ICAM-1) (Squadrito et al.,1997), modification of eNOS activation (Prorock et al., 2003) or estrogen receptormediated events (Pare et al., 2002). We have speculated that indirect estrogen suppression of calpain activation in skeletal muscle also may play a role in inhibition of post-damage neutrophil infiltration (Tiidus et al., 2001; Tiidus, 2003). P/E selectins also may be critical to neutrophil infiltration following skeletal muscle damage (Frenette et al., 2003).

Previous studies also have found differing effects of various forms of muscle damage (i.e., muscle reloading following suspension or eccentric exercise) may induce different regulatory factors and result in differing degrees of neutrophil recruitment (Pizza et al., 2002; Lapointe et al., 2002; Frenette et al., 2003; Tsivitse et al., 2003). Cardiac muscle may also differ from skeletal muscle with regard to mechanisms of post-exercise neutrophil recruitment (Tiidus et al., 2002). Hence, it is possible that estrogen influence on ischemia-induced neutrophil recruitment in skeletal muscle may act through different mechanisms and produce different results than that seen in cardiac muscle or following other forms of muscle damaging injury. These possibilities require further 
investigation. The potential influence of muscle fiber type differences on these factors also requires further clarification.

It also may be possible that at higher physiological concentrations, as found in this experiment, estrogen may act as an indirect pro-oxidant by stimulating the degranulation and release of MPO from neutrophils into plasma (Chiang et al., 2004). In this study, rats were exposed to circulating estrogen levels of around 400$600 \mathrm{pg} / \mathrm{ml}$ of estradiol. This may have been high enough to induce the neutrophil degranulation and MPO loss reported by Chiang and colleagues (2004). Hence, some of the effects of estrogen in attenuating the rise in muscle MPO activities following exercise that were reported in previous studies (i.e., Stupka \& Tiidus, 1999) in which lower physiological levels of estrogen were present (approximately 200$250 \mathrm{pg} / \mathrm{ml}$ estradiol) may have been at least partially negated in this study. If the higher levels of estradiol used in this study did result in partial neutrophil degradulation and loss of MPO into the blood, the MPO levels remaining in neutrophils which infiltrated the skeletal muscle may actually have been lower. Thus, MPO levels measured in this study actually may have represented by default a higher level of muscle neutrophil infiltration, since each neutrophil, now contained less MPO enzymes. This effect could reduce or negate the rise in muscle MPO activities relative to the actual amount of neutrophil infiltration. In addition, it may also be possible that if optimal levels of circulating estrogen are exceeded, its effectiveness in influencing muscle neutrophil infiltration may decline.

Few studies have directly examined the potential for estrogen supplementation to influence indices of muscle disruption following damaging intervention. Komulainen and colleagues (1999) and Amelink and colleagues (1991) have reported female rats as having attenuated indicators of muscle damage following running exercise when compared to male rats. These differences have been attributed to the potential influence of estrogen (Komulainen et al., 1999). However, gender differences in muscle damage indicators have not been demonstrated consistently following exercise in humans (i.e., Stupka et al., 2000; Clarkson and Sayers, 1999). Many (Node et al., 1997; Squadrito et al., 1997; Zhai et al., 2000) but not all (Grist et al., 2002) studies report a reduction on indices of damage with estrogen supplementation following ischemia in rodent cardiac muscle.

This is the first study to report on the effect of estrogen supplementation on indices of skeletal muscle damage following ischemia-induced injury. Muscle G6PD activities (as a marker of pentose phosphate pathway activation) (Armstrong, 1983) and bGLU activities (as a marker of lysosomal enzyme activation) (Komulainen et al., 1999; Salminen and Kihlström, 1985) have been used previously as quantitative markers of muscle damage. In light of the generally positive effects of estrogen supplementation on ischemia-reperfusion injury in cardiac muscle, it is surprising that no such attenuating effect was seen in skeletal muscle G6PD or bGLU activities in any of the groups for up to 72 hours following ischemia in this study. The reasons for the lack of estrogen attenuation of these putatively quantitative indicators of muscle damage are unknown.

It is possible that the mechanisms of damage progression or factors affecting this progression may be influenced by estrogen in cardiac muscle in dissimilar ways or to a greater degree than seen in skeletal muscle. This may also be due to factors such as levels of estrogen receptors or other mechanisms related to damage/ repair or inflammation that could be affected by estrogen, which may differ between skeletal and cardiac muscle (Tiidus et al., 2002). It is known that cardiac muscle contains significantly higher levels of estrogen receptors than skeletal muscle (Lemoine et al., 2002). Hence if the attenuating effect of estrogen on post-ischemic leukocyte infiltration in cardiac muscle is mediated primarily via estrogen receptors, this influence may be less effective in skeletal muscle. The investigation of all of these possibilities exceeds the scope of this study and requires further investigation. 
In conclusion, unlike previous findings following exercise in skeletal muscle or ischemia in cardiac muscle, this study failed to demonstrate estrogen-induced attenuation of indices of neutrophil infiltration or damage in skeletal muscles of male or female rats for up to 72 hours following ischemia. This suggests that estrogen will not act universally to diminish post-damage muscle neutrophil infiltration. This lack of effect may be attributable to possibly distinct effects of different forms of injury on factors regulating muscle inflammatory and damage responses, differences between skeletal and cardiac muscle responses to estrogen and/or to exposure to different estrogen levels. Further research is necessary to further delineate these possibilities.

\section{ACKNOWLEDGMENTS}

This study was supported by a Natural Sciences and Engineering Research Council of Canada (NSERC) Discovery grant to P.M. Tiidus. M. Deller was supported by an NSERC Undergraduate Research Award. M. Gül was supported by a NATO visiting scientist grant administered and awarded by TUBITAK, Turkey.

\section{REFERENCES}

AMELINK GJ, VANDER WAL JH, WOKKE BS, VAN ASBECK J, BAR PR (1991) Exercise-induced muscle damage in the rat: The effect of vitamin E deficiency. Pflügers Arch 419:304-309

ARIANO MA, ARMSTRONG, RB, EDGERTON VR (1973) Hindlimb muscle fiber populations of five mammals. J Histochem Cytochem 21:51-55

ARMSTRONG RB, OGILVIE RW, SCHWANE JA (1983) Eccentric exercise-induced injury to rat skeletal muscle. J Appl Physiol 54:80-93

BAR PR, AMELINK GJ (1997) Protection against muscle damage exerted by estrogen: Hormonal or antioxidant action? Biochem Soc Trans 25:50-54

BELCASTRO AN, ARTHUR GD, ALBISSER TA, RAJ DA (1996) Heart, liver and skeletal muscle myeloperoxidase activity during exercise. J Appl Physiol 80:1331-1335

CHIANG K, PARTHANARATHY S, SANTANAM N (2004) Estrogen, neutrophils and oxidation. Life Sci 75:2425-2438

CLARKSON PM, SAYERS S (1999) Gender differences in exercise-induced muscle damage. In: TARNOPOLSKY MA (ed) Gender Differences in Metabolism. Boca Ratón, FL: CRC Press. pp:283-300
FRENETTE J, CHBINOU N, BODBOUT C MARSOLAIS D, FRENETTE PS (2003) Macrophages, not neutrophils, infiltrate skeletal muscle in mice deficient in $\mathrm{P} / \mathrm{E}$ selectins after mechanical reloading. Am J Physiol Regul Integr Comp Physiol 285:R727-R732

GILLESPIE MJ, GORDON T, MURPHY PR (1987) Motor units and histochemistry in rat lateral gastrocnemius and soleus muscles. J Neurophysiol 57:921-937

GRIST M, WAMBOLT RB, BONDY GP, ENGLISH DR, ALLARD MF (2002) Estrogen replacement stimulates fatty acid oxidation and impairs post-ischemic recovery of hearts from ovariectomized female rats. Can J Physiol Pharmacol 80:1001-1007

KENDALL B, ESTON R (2002) Exercise-induced muscle damage and the potential protective role of estrogen. Sports Med 32:103-123

KOMULAINEN J, KOSKINEN S, KALLIOKOSKI R, TAKALA T, VIHKO V (1999) Gender differences in skeletal muscle fibre damage after eccentrically biased downhill running in rats. Acta Physiol Scand $165: 57-63$

LA PONTE BM, FRENETTE J, COTE CH (2002) Lengthening contraction-induced inflammation is linked to secondary damage but devoid of neutrophil invasion. J Appl Physiol 92:1995-2004

LEMOINE S, GRANIER P TIFFOCHE C, BERTHON P, RANNOU-BEKONO F THIEULANT ML, CARRE F, DELAMARCHE P (2002) Effect of endurance training on oestrogen receptor alpha transcripts in rat skeletal muscle. Acta Physiol Scand 174:283-289

LOWRY OH, ROSENBROUGH N, LEWIS A, RANDALL $\mathrm{R}$ (1951) Protein measurements with Folin phenol reagent. J Biol Chem 193:265-270

NILSSON S, MAKELA S, TREUTER, E. TUJAGUE M, THOMSEN J, ANDERSSON G, ENMARK E, PETTERSSON K, WARNER M, GUSTAFSSON JA (2001) Mechanisms of estrogen action. Physiol Rev $81: 1535-1565$

NODE K, KITAKAZE M, KOSAKA H, MINOMINO T, FUNAYA H, HORI M (1997) Amelioration of ischemia- and reperfusion-induced myocardial injury by 17ß-estradiol. Circulation 96:1953-1963

PARE G, KRUST A, KARAS RH, DUPONT S, ARONOVITZ M, CHAMBON P, MENDELSOHN ME (2002) Estrogen receptor- $\alpha$ mediates the protective effects of estrogen against vascular injury. Circ Res 90:1087-1092

PIZZA FX, KOH TJ, MCGREGOR SJ, BROOKS SV (2002) Muscle inflammatory cells after passive stretches, isometric contractions, and lengthening contractions. J Appl Physiol 92:1873-1878

PROROCK AJ, HAFEZI-MOGHADAM A, LAUBACH VE, LIAO JK, LEY K (2003) Vascular protection by estrogen in ischemia-reperfusion injury requires endothelial nitric oxide synthase. Am J Physiol Heart Circ Physiol 284:H133-H140

RUBIN BB, ROMASCHIN A, WALKER, PM, GUTE D, KORTHUIS RJ (1996) Mechanisms of postischemic injury in skeletal muscle: Intervention strategies. J Appl Physiol 80:369-387

SALMINEN A, KIHLSTROM M (1985) Lysosomal changes in mouse skeletal muscle during repair of exercise injuries. Muscle Nerve 8:269-279

SARWAR R, NICLOS BB, RUTHERFORD OM (1996) Changes in muscle strength, relaxation rate, and fatiguability during the human menstrual cycle. J Physiol 493:267-272

SCHLAG MG, HARRIS KA, POTTER RF (2001) Role of leukocyte accumulation and oxygen radicals in 
ischemia-reperfusion-induced injury in skeletal muscle. Am J Physiol Heart Circ Physiol 280:H1716H1721

SMITH JK, GRISHAM MB, GRANGER DN, KORTHUIS RJ (1989) Free radical defense mechanisms and neutrophil infiltration in postischemic skeletal muscle. Am J Physiol 256:H789-H793

SQUADRITO F, ALTAVILLA D, SQUADRITO G, CAMPO, G, ARLOTTA M, ARCORACI V, MINUTOLI L, SERRANO M, SAITTA A, CAPUTI AP (1997) 17B-oestradiol reduces cardiac leukocyte accumulation in myocardial ischaemia reperfusion injury in rat. Eur J Pharmacol 355:185-192

STUPKA N, LOWTHER S, CHORNEYKO K, BOURGEOIS J, HOGBEN C, TARNOPOLSKY MA (2000) Gender differences in muscle inflammation following eccentric exercise. J Appl Physiol 89:23252332

STUPKA N, TIIDUS PM (2001) Effects of ovariectomy and estrogen on ischemia-reperfusion injury in hindlimbs of female rats. J Appl Physiol 91:1828-1835

TIDBALL J, BERCHENKO E, FRENETTE J (1999) Macrophage invasion does not contribute to muscle membrane injury during inflammation. J Leukocyte Biol 65:492-498
TIIDUS PM (2003) Influence of estrogen on skeletal muscle damage, inflammation and repair. Exerc Sports Sci Rev 31:40-44

TIIDUS PM. BOMBARDIER E (1999) Oestrogen attenuates post-exercise myeloperoxidase activity in skeletal muscle of male rats. Acta Physiol Scand 166:85-90

TIIDUS PM, HOLDEN D, BOMBARDIER E, ZAJCHOWSKI S, ENNS D, BELCASTRO A (2001) Estrogen effect on post-exercise skeletal muscle neutrophil infiltration and calpain activity. Can $\mathrm{J}$ Physiol Pharmacol 79:400-406

TIIDUS, PM, ZAJCHOWSKI S. ENNS D, HOLDEN D, BOMBARDIER E, BELCASTRO A (2002) Differential effect of oestrogen on post-exercise cardiac muscle myeloperoxidase and calpain activities in female rats. Acta Physiol Scand 174:131-136

TSIVITSE SK, MCLOUGHLIN T, PETERSON J, MYLONA E, MCGREGOR S, PIZZA FX (2003) Downhill running in rats: Influence on neutrophils, macrophages and MyoD+ cells in skeletal muscle. Eur J Appl Physil 90:633-638

ZHAI P, EURELL TE, COOLE P, LUBAHN D, GROSS DR (2000) Myocardial ischemia-reperfusion injury in estrogen receptor- $\alpha$ knockout and wild-type mice. Am J Physiol Heart Circ Physiol 278:H1640-H1647 